\title{
A GENERALISATION OF THE ERDÉLYI-KOBER OPERATORS
}

\author{
by J. S. LOWNDES \\ (Received 10th October 1969)
}

\section{Introduction}

In this paper we introduce a generalised Hankel operator and generalised Erdélyi-Kober operators and deduce some relations between them. The operators are then applied to obtain solutions to some dual integral equations which have applications in diffraction theory.

The analysis throughout is formal and many of the results which we require, apart from those listed below, can be found in the book by Sneddon (2). Indeed a comparison of the subsequent sections with the sections (2.1), (2.4), (2.5), (4.2) and (4.3) of (2) will show how closely the work in this paper resembles the development and application of the Erdelyi-Kober operators as described by Sneddon.

Burlak (1) has shown that if $-1<\beta<0$, then the solutions of the integral equations

$$
\int_{0}^{x} \dot{\psi}(y)\left(x^{2}-y^{2}\right)^{\frac{1}{2} \beta} J_{\beta}\left\{k \sqrt{ }\left(x^{2}-y^{2}\right)\right\} d y=h(x)
$$

and

$$
\int_{x}^{\infty} \psi(y)\left(y^{2}-x^{2}\right)^{\frac{1}{2} \beta} J_{\beta}\left\{k \sqrt{ }\left(y^{2}-x^{2}\right)\right\} d y=m(x)
$$

are given by

$$
\psi(x)=k \frac{d}{d x} \int_{0}^{x} y h(y)\left(x^{2}-y^{2}\right)^{-\frac{1}{(\beta+1)} I_{-(\beta+1)}\left\{k \sqrt{ }\left(x^{2}-y^{2}\right)\right\} d y}
$$

and

$$
\psi(x)=-k \frac{d}{d x} \int_{x}^{\infty} y m(y)\left(y^{2}-x^{2}\right)^{-\frac{1}{2(\beta+1)} I_{-(\beta+1)}}\left\{k \sqrt{ }\left(y^{2}-x^{2}\right)\right\} d y
$$

respectively. If $J_{\beta}$, the Bessel function of the first kind, is replaced by $I_{\beta}$, the modified Bessel function of the first kind, in equations (1) and (2), then $I_{-(\beta+1)}$ must be replaced by $J_{-(\beta+1)}$ in equations (3) and (4). The condition $-1<\beta<0$ was not stated by Burlak but was pointed out in a more recent paper by Srivastav (3). 


\section{The generalised operator of the Hankel transform}

We introduce the operator defined by

$$
\begin{aligned}
& S\left(\begin{array}{ll}
a, b, k \\
\eta, \alpha, \sigma
\end{array}\right) f(x)=2^{\alpha} x^{2 \sigma-\alpha}\left(x^{2}-a^{2}\right)^{-\sigma} \\
& \qquad \int_{k}^{\infty} u^{1-2 \sigma-\alpha}\left(u^{2}-k^{2}\right)^{\sigma} f(u) J_{2 \eta+\alpha}\left\{\sqrt{ }\left[\left(x^{2}-a^{2}\right)\left(u^{2}-b^{2}\right)\right]\right\} d u,
\end{aligned}
$$

which is related to the modified Hankel operator $S_{\eta, \alpha}$ defined in (2, p. 30), by

$$
S\left(\begin{array}{lll}
0, & 0,0 \\
\eta, & \alpha, \sigma
\end{array}\right)=S\left(\begin{array}{lll}
0, & 0,0 \\
\eta, & \alpha, & 0
\end{array}\right)=S_{\eta, \alpha}
$$

Applying the Hankel inversion theorem (2, p. 29) to the equation

we find that

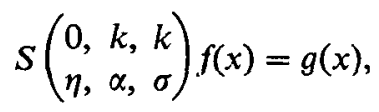

$$
f(u)=S\left(\begin{array}{lll}
k, & 0, & 0 \\
\eta+\alpha, & -\alpha, & \sigma
\end{array}\right) g(u)
$$

and hence an inversion theorem for the generalised operator of the Hankel transform can be written in the form

$$
S^{-1}\left(\begin{array}{lll}
0, & k, k \\
\eta, & \alpha, & \sigma
\end{array}\right)=S\left(\begin{array}{lll}
k, & 0 . & 0 \\
\eta+\alpha, & -\alpha, & \sigma
\end{array}\right)
$$

When $k=0$ we see that the above equation becomes

$$
S_{\eta, \alpha}^{-1}=S_{\eta+\alpha,-\alpha}
$$

which agrees with the result given in (2, p. 30).

\section{The generalised Erdélyi-Kober operators}

We define the operators $\Im_{k}(\eta, \alpha)$ and $\mathfrak{S}_{k}(\eta, \alpha)$ by the formulae

$\mathfrak{I}_{k}(\eta, \alpha) f(x)=2^{\alpha} x^{-2 \eta-2 \alpha} k^{1-\alpha} \int_{0}^{x} u^{1+2 \eta}\left(x^{2}-u^{2}\right)^{\frac{1}{2(\alpha-1)}} J_{\alpha-1}\left\{k \sqrt{ }\left(x^{2}-u^{2}\right)\right\} f(u) d u$,

$\mathcal{R}_{k}(\eta, \alpha) f(x)=2^{x} x^{2 \eta} k^{1-\alpha} \int_{x}^{\infty} u^{1-2 \eta-2 \alpha}\left(u^{2}-x^{2}\right)^{\frac{1}{2(\alpha-1)}} J_{\alpha-1}\left\{k \sqrt{ }\left(u^{2}-x^{2}\right)\right\} f(u) d u$,

where $\alpha>0, \eta>-\frac{1}{2}$, and the operators $\mathfrak{I}_{i k}(\eta, \alpha)$ and $\boldsymbol{R}_{i k}(\eta, \alpha)$ by the above equations when $J_{\alpha-1}$ is replaced by $I_{\alpha-1}$. Similar formulae have been briefly discussed by Srivastav (3).

We shall make use of some of the basic properties of the operators whose derivation we shall now indicate. 
If we let $k$ tend to zero we see that these operators are related to the Erdélyi-Kober operators (2, p. 48) by

$$
\Im_{0}(\eta, \alpha)=I_{\eta, \alpha}, \quad \Omega_{0}(\eta, \alpha)=K_{\eta, \alpha} .
$$

Letting $\alpha$ tend to zero in equations (10) we have the identity operators

$$
\mathfrak{I}_{0}(\eta, 0)=I_{\eta, 0}=I, \quad \Re_{0}(\eta, 0)=K_{\eta, 0}=I .
$$

From the definitions (8) and (9) it follows immediately that

$$
\begin{aligned}
& \Im_{k}(\eta, \alpha) x^{2 \beta} f(x)=x^{2 \beta} \Im_{k}(\eta+\beta, \alpha) f(x), \\
& \Re_{k}(\eta, \alpha) x^{2 \beta} f(x)=x^{2 \beta} \Re_{k}(\eta-\beta, \alpha) f(x) .
\end{aligned}
$$

Writing down the expressions for $\mathfrak{I}_{i k}(\eta+\alpha, \beta)$ and $\mathfrak{I}_{k}(\eta, \alpha)$ we find that

$$
\begin{aligned}
\mathfrak{I}_{i k}(\eta+\alpha, \beta) \mathfrak{I}_{k}(\eta, \alpha) f(x) & =2^{\alpha+\beta} x^{-2(\eta+\alpha+\beta)} k^{2-\alpha-\beta} \\
& \int_{0}^{x} u\left(x^{2}-u^{2}\right)^{\frac{1}{(\beta-1)}} I_{\beta-1}\left\{k \sqrt{ }\left(x^{2}-u^{2}\right)\right\} d u \\
& \int_{0}^{u} y^{1+2 \eta}\left(u^{2}-y^{2}\right)^{\frac{t(\alpha-1)}{(\alpha)}} J_{\alpha-1}\left\{k \sqrt{ }\left(u^{2}-y^{2}\right)\right\} f(y) d y .
\end{aligned}
$$

Interchanging the order of the integrations and evaluating the inner integral $(2$, p. 31, (2.1.34)) we get

$$
\begin{aligned}
\mathfrak{I}_{i k}(\eta+\alpha, \beta) \mathfrak{I}_{k}(\eta, \alpha) f(x) & \\
& =\frac{2}{\Gamma(\alpha+\beta)} x^{-2(\eta+\alpha+\beta)} \int_{0}^{x} y^{1+2 \eta}\left(x^{2}-y^{2}\right)^{\alpha+\beta-1} f(y) d y \\
& =\mathfrak{I}_{0}(\eta, \alpha+\beta) f(x)=I_{\eta, \alpha+\beta} f(x),
\end{aligned}
$$

so that we have the product rule

$$
\mathfrak{I}_{i k}(\eta+\alpha, \beta) \mathfrak{I}_{k}(\eta, \alpha)=I_{\eta, \alpha+\beta} .
$$

In a similar way we can derive the formulae

$$
\mathfrak{I}_{k}(\eta+\alpha, \beta) \mathfrak{I}_{i k}(\eta, \alpha)=I_{\eta, \alpha+\beta}
$$

and

$$
\mathfrak{R}_{i k}(\eta, \alpha) \mathfrak{R}_{k}(\eta+\alpha, \beta)=\mathfrak{\Re}_{k}(\eta, \alpha) \mathfrak{S}_{i k}(\eta+\alpha, \beta)=K_{\eta, \alpha+\beta} .
$$

The above results indicate the manner in which we should define the operators $\mathfrak{I}_{k}(\eta, \alpha)$ and $\boldsymbol{\Omega}_{k}(\eta, \alpha)$ for $\alpha<0$. From equations (11) and (13) we have

$$
\mathfrak{I}_{i k}(\eta+\alpha,-\alpha) \mathfrak{I}_{k}(\eta, \alpha)=I,
$$

which suggests that if $\alpha<0$ we define $g=\mathfrak{I}_{k}(\eta, \alpha) f$ to be the solution of the 
integral equation

$$
\begin{aligned}
& \mathfrak{I}_{i k}(\eta+\alpha,-\alpha) g(x) \\
& =2^{-\alpha} x^{-2 \eta} k^{1+\sigma} \int_{0}^{x} u^{1+2 \eta+2 \alpha}\left(x^{2}-u^{2}\right)^{-\frac{1}{2}(\alpha+1)} I_{-(\alpha+1)}\left\{k \sqrt{ }\left(x^{2}-u^{2}\right)\right\} g(u) d u=f(x) .
\end{aligned}
$$

Using the results (1) and (3) it follows that $\mathfrak{I}_{k}(\eta, \alpha) f$ is given by the equation

$$
\begin{aligned}
\Im_{k}(\eta, \alpha) f(x) & =2^{\alpha} x^{-1-2 \eta-2 \alpha} k^{-\alpha} \frac{d}{d x} \int_{0}^{x} u^{1+2 \eta}\left(x^{2}-u^{2}\right)^{\frac{1}{a} \alpha} J_{\alpha}\left\{k \sqrt{ }\left(x^{2}-u^{2}\right)\right\} f(u) d u \\
& =x^{-1-2 \eta-2 \alpha} \mathscr{D}_{x}\left\{x^{3+2 \eta+2 \alpha} \mathfrak{J}_{k}(\eta, \alpha+1) f(x)\right\}
\end{aligned}
$$

when $-1<\alpha<0$ and where we have written $\mathscr{D}_{x}=\frac{1}{2} \frac{d}{d x} x^{-1}$.

Similarly from equations (11) and (15) we see that if we define $g=\Omega_{k}(\eta, \alpha) f$ to be the solution of the integral equation $\Omega_{i k}(\eta+\alpha,-\alpha) g=f$, and use the results (2) and (4), then

$$
\begin{aligned}
\mathfrak{S}_{k}(\eta, \alpha) f(x) & =-2^{\alpha} x^{2 \eta-1} k^{-\alpha} \frac{d}{d x} \int_{x}^{\infty} u^{1-2 \eta-2 \alpha}\left(u^{2}-x^{2}\right)^{\frac{1}{2} \alpha} J_{\alpha}\left\{k \sqrt{ }\left(u^{2}-x^{2}\right)\right\} f(u) d u \\
& =-x^{2 \eta-1} \mathscr{D}_{x}\left\{x^{3-2 \eta} \mathcal{S}_{k}(\eta-1, \alpha+1) f(x)\right\},
\end{aligned}
$$

where $-1<\alpha<0$.

Using a similar method to that employed in $(2$, p. 51$)$ we can show that when $\alpha<0$ general expressions for the operators are

$$
\mathfrak{I}_{k}(\eta, \alpha) f(x)=x^{-1-2 \eta-2 \alpha} \mathscr{D}_{x}^{m}\left\{x^{2 m+1+2 \alpha+2 \eta} \mathfrak{I}_{k}(\eta, \alpha+m) f(x)\right\}
$$

and

$$
\mathfrak{\Re}_{k}(\eta, \alpha) f(x)=(-1)^{m} x^{2 \eta-1} \mathscr{D}_{x}^{m}\left\{x^{2 m+1-2 \eta} \mathfrak{\Re}_{k}(\eta-m, \alpha+m) f(x)\right\},
$$

where $-m<\alpha<0$ and $m$ is a positive integer.

Now that we have defined the operators for negative $\alpha$ we see that equations (11), (13), (14) and (15) can be interpreted as yielding the inverse operators

$$
\begin{array}{ll}
\mathfrak{I}_{i k}^{-1}(\eta, \alpha)=\mathfrak{I}_{k}(\eta+\alpha,-\alpha), & \mathfrak{I}_{k}^{-1}(\eta, \alpha)=\mathfrak{I}_{i k}(\eta+\alpha,-\alpha), \\
\mathfrak{R}_{i k}^{-1}(\eta, \alpha)=\mathfrak{\Re}_{k}(\eta+\alpha,-\alpha), & \mathfrak{R}_{k}^{-1}(\eta, \alpha)=\mathfrak{\Re}_{i k}(\eta+\alpha,-\alpha) .
\end{array}
$$

Finally it is an easy matter to show that

$$
\int_{0}^{\infty} x f(x) \mathfrak{I}_{k}(\eta, \alpha) g(x) d x=\int_{0}^{\infty} x g(x) \mathfrak{S}_{k}(\eta, \alpha) f(x) d x
$$




\section{Relations between the generalised Hankel and the generalised Erdélyi-Kober operators}

From the definitions (5) and (8) we have that

$$
\begin{aligned}
\Im_{i k}(\eta+\alpha, \beta) S\left(\begin{array}{ll}
0,0, k \\
\eta, \alpha, \sigma
\end{array}\right) f(x)= & 2^{\alpha+\beta} x^{-2 \eta-2 \alpha-2 \beta} k^{1-\beta} \\
& \int_{0}^{x} u^{1+2 \eta+\alpha}\left(x^{2}-u^{2}\right)^{\ddagger(\beta-1)} I_{\beta-1}\left\{k \sqrt{\left.\left(x^{2}-u^{2}\right)\right\} d u}\right. \\
& \int_{k}^{\infty} y^{1-2 \sigma-\alpha}\left(y^{2}-k^{2}\right)^{\sigma} J_{2 \eta+\alpha}(u y) f(y) d y .
\end{aligned}
$$

Interchanging the order of the integrations and evaluating the inner integral using the result $(2$, p. $31,(2.1 .34))$, we get

$$
\begin{aligned}
& \mathfrak{I}_{i k}(\eta+\alpha, \beta) S\left(\begin{array}{ll}
0,0, k \\
\eta, \alpha, \sigma
\end{array}\right) f(x) \\
& =2^{\alpha+\beta} x^{-\alpha-\beta} \int_{k}^{\infty} y^{1+2 \eta-2 \sigma}\left(y^{2}-k^{2}\right)^{\sigma-\lambda} J_{2 \lambda}\left\{x \sqrt{ }\left(y^{2}-k^{2}\right)\right\} f(y) d y
\end{aligned}
$$

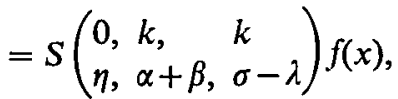

where $2 \lambda=2 \eta+\alpha+\beta$.

In this way we have established the relation

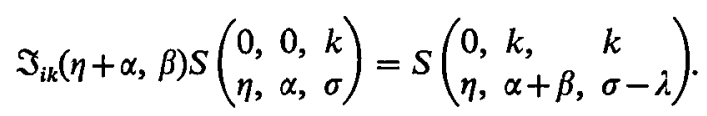

Using the result (2, p. 31, (2.1.31)) we can, by a similar method, show that

$$
\Re_{k}(\eta, \alpha) S\left(\begin{array}{ll}
0, & 0, k \\
\eta+\alpha, & \beta, \sigma
\end{array}\right)=S\left(\begin{array}{lll}
0, & k, & k \\
\eta, & \alpha+\beta, & \sigma+\lambda
\end{array}\right), \quad 2 \lambda=2 \eta+\alpha+\beta .
$$

By a similar process we can also establish the relations

and

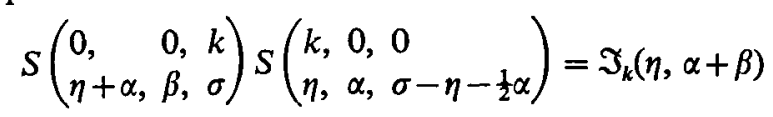

$$
S\left(\begin{array}{ll}
0, & 0,0 \\
\eta, & \alpha, 0
\end{array}\right) S\left(\begin{array}{ll}
k, & 0,0 \\
\eta+\alpha, & \beta, \eta+\alpha+\frac{1}{2} \beta
\end{array}\right)=\mathfrak{R}_{i k}(\eta, \alpha+\beta) .
$$

\section{Solution of the dual integral equations}

The dual integral equations

$$
\begin{gathered}
\int_{k}^{\infty} u^{-\mu-v}\left(u^{2}-k^{2}\right)^{\beta} \psi(u) J_{\mu}(x u) d u=F_{1}(x), \quad 0 \leqq x<1, \quad k \geqq 0, \\
\int_{0}^{\infty} \psi(u) J_{v}(x u) d u=G_{2}(x), \quad x>1,
\end{gathered}
$$


where $F_{1}(x)$ and $G_{2}(x)$ are prescribed functions, have been solved by Burlak (1) using a generalisation of the method introduced by Sneddon $(2$, p. 74$)$ and developed by Copson (2, p. 91) for solving the case $\mu=v, k=0$.

Following Sneddon we use the notation $I_{1}=\{x: 0 \leqq x<1\}, I_{2}=\{x: x>1\}$ and write any function $f(x), x \geqq 0$, as

where

$$
f(x)=f_{1}(x)+f_{2}(x)
$$

$$
f_{1}(x)=\left\{\begin{array}{ll}
f(x), & x \in I_{1}, \\
0, & x \in I_{2},
\end{array} \quad f_{2}(x)= \begin{cases}0, & x \in I_{1}, \\
f(x), & x \in I_{2} .\end{cases}\right.
$$

If we make the substitutions

$$
\psi(u)=u^{1+v} \phi(u), \quad f(x)=2^{\mu-2 \beta} x^{2 \beta-\mu} F(x), \quad g(x)=2^{-v} x^{v} G(x),
$$

we see that equations (30) and (31) can be written in the operator form

$$
\begin{aligned}
& S\left(\begin{array}{lll}
0, & 0, & k \\
\beta, & \mu-2 \beta, & \beta
\end{array}\right) \phi(x)=f(x), \\
& S\left(\begin{array}{lll}
0, & 0, & 0 \\
v, & -v, & 0
\end{array}\right) \phi(x)=g(x),
\end{aligned}
$$

where $\phi(x), f_{2}(x)$ and $g_{1}(x)$ are unknown and the functions

are given.

$$
f_{1}(x)=2^{\mu-2 \beta} x^{2 \beta-\mu} F_{1}(x), \quad g_{2}(x)=2^{-v} x^{\nu} G_{2}(x)
$$

\subsection{First method of solution}

It follows from the results (26) and (27) that

$$
\begin{aligned}
\Im_{i k}(\mu-\beta, \beta-\mu) S & \left(\begin{array}{lll}
0, & 0, & k \\
\beta, & \mu-2 \beta, & \beta
\end{array}\right) \\
& =\Re_{k}(\beta, \nu-\beta) S\left(\begin{array}{lll}
0, & 0, & 0 \\
\nu, & -v, & 0
\end{array}\right)=S\left(\begin{array}{lll}
0, & k, & k \\
\beta, & -\beta, & \frac{1}{2} \beta
\end{array}\right),
\end{aligned}
$$

and hence we can write equations (33) and (34) in the form

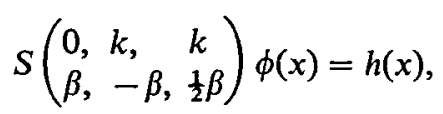

where

$$
h_{1}(x)=\mathfrak{I}_{i k}(\mu-\beta, \beta-\mu) f_{1}(x), \quad h_{2}(x)=\mathfrak{\Omega}_{k}(\beta, \nu-\beta) g_{2}(x)
$$

are known functions.

Applying the inversion formula (7) to equation (37) we see that

$$
\phi(u)=S^{-1}\left(\begin{array}{lll}
0, & k, & k \\
\beta, & -\beta, & \frac{1}{2} \beta
\end{array}\right) h(u)=S\left(\begin{array}{lll}
k, & 0, & 0 \\
0, & \beta, & \frac{1}{2} \beta
\end{array}\right) h(u) .
$$

Reverting to the original variables (32) and making use of the formulae (12) 
we find that the solution of the dual integral equations can be written as

$$
\psi(u)=u^{1+v} \phi(u)=u^{1+v} S\left(\begin{array}{lll}
k, & 0, & 0 \\
0, & \beta, & \frac{1}{2} \beta
\end{array}\right) h(u)
$$

which is

$\psi(u)=2^{\beta} u^{1+v}\left(u^{2}-k^{2}\right)^{-\frac{1}{2} \beta}\left\{\int_{0}^{1} h_{1}(x)+\int_{1}^{\infty} h_{2}(x)\right\} x^{1-\beta} J_{\beta}\left\{x \sqrt{ }\left(u^{2}-k^{2}\right)\right\} d x$,

where

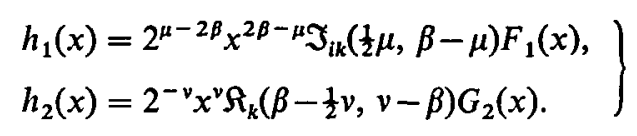

We consider four cases.

(i) When $v>\beta>\mu>-1$.

Using the definitions (8) and (9) we see that equations (42) are

$$
\begin{aligned}
& h_{1}(x)=2^{-\beta} k^{1+\mu-\beta} \int_{0}^{x} t^{1+\mu}\left(x^{2}-t^{2}\right)^{\frac{1}{2(\beta-\mu-1)}} I_{\beta-\mu-1}\left\{k \sqrt{ }\left(x^{2}-t^{2}\right)\right\} F_{1}(t) d t, \\
& h_{2}(x)=2^{-\beta} k^{1+\beta-v} x^{2 \beta} \int_{x}^{\infty} t^{1-v}\left(t^{2}-x^{2}\right)^{\frac{1}{2}(v-\beta-1)} J_{\nu-\beta-1}\left\{k \sqrt{ }\left(t^{2}-x^{2}\right)\right\} G_{2}(t) d t,
\end{aligned}
$$

which together with equation (41) furnish a solution to the dual integral equations.

(ii) When $1+\beta>\mu>\beta>v>\beta-1$.

Applying the definitions (18) and (19) we find that equations (42) become

$$
\begin{array}{r}
h_{1}(x)=2^{-\beta} x^{-1} k^{\mu-\beta} \frac{d}{d x} \int_{0}^{x} t^{1+\mu}\left(x^{2}-t^{2}\right)^{\frac{1}{2(\beta-\mu)}} I_{\beta-\mu}\left\{k \sqrt{ }\left(x^{2}-t^{2}\right)\right\} F_{1}(t) d t,(46) \\
h_{2}(x)=-2^{-\beta} x^{2 \beta-1} k^{\beta-v} \frac{d}{d x} \int_{x}^{\infty} t^{1-v}\left(t^{2}-x^{2}\right)^{\frac{1}{2(v-\beta)}} J_{v-\beta}\left\{k \sqrt{ }\left(t^{2}-x^{2}\right)\right\} G_{2}(t) d t \text {. (47) }
\end{array}
$$

The solution to the dual integral equations, given by equations (41), (46) and (47), is in complete agreement with that obtained by Burlak.

(iii) When $v=\mu, \beta-1<\mu<\beta$.

In this case $\psi(u)$ is given by equation (41) with $v=\mu, h_{1}(x)$ by equation (43) and $h_{2}(x)$ by equation (47) with $v=\mu$.

(iv) When $v=\mu, \beta<\mu<\beta+1$.

Here $\psi(u)$ is given by equation (41) with $v=\mu, h_{1}(x)$ by equation (46) and $h_{2}(x)$ is given by equation (44) with $v=\mu$.

$$
\text { E.M.S. }-\mathrm{K}
$$


It is perhaps of interest to note that if, in the solutions (iii) and (iv), we write $\beta=\mu-\alpha$ and let $k$ tend to zero, we obtain solutions to the equations

$$
\begin{gathered}
\int_{0}^{\infty} u^{-2 \alpha} \psi(u) J_{\mu}(x u) d u=F_{1}(x), \quad 0 \leqq x<1, \\
\int_{0}^{\infty} \psi(u) J_{\mu}(x u) d u=G_{2}(x), \quad x>1,
\end{gathered}
$$

valid for $-1<\alpha<0$ and $0<\alpha<1$ respectively, which are in agreement with those given in (2, pp. 86-87).

\subsection{Second method of solution (Burlak's method)}

We regard the function

$$
\phi(u)=S\left(\begin{array}{lll}
k, & 0,0 \\
0, & \beta, \frac{1}{2} \beta
\end{array}\right) h(u)
$$

as a trial solution of equations (33) and (34) where $h(u)$ is a function as yet undetermined.

Substituting this expression for $\phi(u)$ in equations (33) and (34) and using the formulae (28) and (29) we get

$$
\begin{aligned}
& f(x)=S\left(\begin{array}{lll}
0, & 0, & k \\
\beta, & \mu-2 \beta, & \beta
\end{array}\right) S\left(\begin{array}{lll}
k, & 0, & 0 \\
0, & \beta, & \frac{1}{2} \beta
\end{array}\right) h=\Im_{k}(0, \mu-\beta) h(x), \\
& g(x)=S\left(\begin{array}{lll}
0, & 0, & 0 \\
v, & -v, & 0
\end{array}\right) S\left(\begin{array}{lll}
k, & 0, & 0 \\
0, & \beta, & \frac{1}{2} \beta
\end{array}\right) h=\Re_{i k}(v, \beta-v) h(x) .
\end{aligned}
$$

Making use of the inverse operators (22) and (23) we obtain the following expressions for the functions $h_{1}(x)$ and $h_{2}(x)$

$$
\begin{aligned}
& h_{1}(x)=\mathfrak{I}_{k}^{-1}(0, \mu-\beta) f_{1}(x)=\mathfrak{I}_{i k}(\mu-\beta, \beta-\mu) f_{1}(x), \\
& h_{2}(x)=\mathfrak{\Re}_{i k}^{-1}(\nu, \beta-v) g_{2}(x)=\mathfrak{I}_{k}(\beta, v-\beta) g_{2}(x) .
\end{aligned}
$$

The expression for $\phi(u)$ is then given by equations (48), (51) and (52) and this is identical with the solution found by the first method.

\subsection{Functions derived from the solutions of the integral equations}

In many mixed boundary value problems the quantities of interest are not only the solution $\psi(u)$ of equations (30) and (31) but also the functions $F_{2}(x)$ and $G_{1}(x)$ defined by these equations for the ranges $x>1$ and $0 \leqq x<1$, respectively. We now indicate a method of determining these functions in terms of the known functions $F_{1}(x)$ and $G_{2}(x)$. 

that

In terms of the original variables (32) we see from equations (49) and (50)

$$
\begin{aligned}
& F(x)=2^{2 \beta-\mu} x^{\mu-2 \beta} \mathfrak{I}_{k}(0, \mu-\beta) h(x), \\
& G(x)=2^{v} x^{-v} \Re_{i k}(v, \beta-v) h(x),
\end{aligned}
$$

where the functions $h_{1}(x)$ and $h_{2}(x)$ are given by equations (42).

We shall distinguish two cases.

(i) When $1+\beta>v>\beta>\mu>\beta-1$.

In this case equations (42) become equations (43) and (44) and can be written in the form

$h_{1}(x)=2^{-\beta} k^{1+\mu-\beta} F_{1}^{*}(x) H(1-x), \quad h_{2}(x)=2^{-\beta} x^{2 \beta} k^{1+\beta-\gamma} G_{2}^{*}(x) H(x-1)$,

where $H(x)$ is the Heaviside unit function and

$$
\begin{aligned}
& F_{1}^{*}(x)=\int_{0}^{x} t^{1+\mu}\left(x^{2}-t^{2}\right)^{ \pm(\beta-\mu-1)} I_{\beta-\mu-1}\left\{k \sqrt{ }\left(x^{2}-t^{2}\right)\right\} F_{1}(t) d t, \\
& G_{2}^{*}(x)=\int_{x}^{\infty} t^{1-\nu}\left(t^{2}-x^{2}\right)^{ \pm(\nu-\beta-1)} J_{\nu-\beta-1}\left\{k \sqrt{ }\left(t^{2}-x^{2}\right)\right\} G_{2}(t) d t .
\end{aligned}
$$

From equation (53) we have

$$
\begin{array}{r}
F(x)=k x^{-\mu-1} \frac{d}{d x}\left\{\int_{0}^{x} F_{1}^{*}(u) H(1-u)+k^{2 \beta-v-\mu} \int_{0}^{x} u^{2 \beta} G_{2}^{*}(u) H(u-1)\right\} \\
u\left(x^{2}-u^{2}\right)^{\frac{1}{(\mu-\beta)} J_{\mu-\beta}}\left\{k \sqrt{ }\left(x^{2}-u^{2}\right)\right\} d u,
\end{array}
$$

and hence

$$
\begin{aligned}
F_{2}(x)=k x^{-\mu-1} \frac{d}{d x}\left\{\int_{0}^{1} F_{1}^{*}(u)+k^{2 \beta-v-\mu} \int_{1}^{x} u^{2 \beta} G_{2}^{*}(u)\right\} \\
u\left(x^{2}-u^{2}\right)^{\frac{1}{2}(\mu-\beta)} J_{\mu-\beta}\left\{k \sqrt{ }\left(x^{2}-u^{2}\right)\right\} d u .
\end{aligned}
$$

In a similar way from equation (54) we obtain

$$
\begin{aligned}
G_{1}(x)=-k x^{\nu-1} \frac{d}{d x}\left\{k^{v+\mu-2 \beta} \int_{x}^{1} u^{-2 \beta} F_{1}^{*}(u)+\int_{1}^{\infty} G_{2}^{*}(u)\right\} \\
u\left(u^{2}-x^{2}\right)^{\frac{1}{2(\beta-v)} I_{\beta-v}}\left\{k \sqrt{ }\left(u^{2}-x^{2}\right)\right\} d u .
\end{aligned}
$$

Equations (58) and (59) determine the functions $F_{2}(x)$ and $G_{1}(x)$ in terms of the known functions $F_{1}^{*}(x)$ and $G_{2}^{*}(x)$ which are given by equations $(56)$ and (57) respectively.

(ii) When $1+\beta>\mu>\beta>v>\beta-1$.

The functions $h_{1}(x)$ and $h_{2}(x)$ are given by equations (46) and (47) which we write in the form

$$
\begin{aligned}
& h_{1}(x)=2^{-\beta} x^{-1} k^{\mu-\beta} F_{1}^{* *}(x) H(1-x), \\
& h_{2}(x)=-2^{-\beta} x^{2 \beta-1} k^{\beta-v} G_{2}^{* *}(x) H(x-1),
\end{aligned}
$$


where

$$
\begin{aligned}
& F_{1}^{* *}(x)=\frac{d}{d x} \int_{0}^{x} t^{1+\mu}\left(x^{2}-t^{2}\right)^{\frac{t}{t(\beta-\mu)}} I_{\beta-\mu}\left\{k \sqrt{ }\left(x^{2}-t^{2}\right)\right\} F_{1}(t) d t \\
& G_{2}^{* *}(x)=\frac{d}{d x} \int_{x}^{\infty} t^{1-\nu}\left(t^{2}-x^{2}\right)^{\frac{1}{2}(\nu-\beta)} j_{\nu-\beta}\left\{k \sqrt{ }\left(t^{2}-x^{2}\right)\right\} G_{2}(t) d t .
\end{aligned}
$$

From equations (53) and (54) it is easily shown that in this case we get

$$
\begin{aligned}
& F_{2}(x)=k x^{-\mu}\left\{\int_{0}^{1} F_{1}^{* *}(u)-k^{2 \beta-\nu-\mu} \int_{1}^{x} u^{2 \beta} G_{2}^{* *}(u)\right\} \\
&\left(x^{2}-u^{2}\right)^{\frac{1}{2}(\mu-\beta-1)} J_{\mu-\beta-1}\left\{k \sqrt{ }\left(x^{2}-u^{2}\right)\right\} d u, \\
& G_{1}(x)=k x^{v}\left\{k^{\nu+\mu-2 \beta} \int_{x}^{1} u^{-2 \beta} F_{1}^{* *}(u)-\int_{1}^{\infty} G_{2}^{* *}(u)\right\} \\
&\left(u^{2}-x^{2}\right)^{\frac{z}{2}(\beta-\nu-1)} I_{\beta-v-1}\left\{k \sqrt{ }\left(u^{2}-x^{2}\right)\right\} d u .
\end{aligned}
$$

The results (63) and (64) are exactly the same as those given by Burlak.

\section{REFERENCES}

(1) J. BuRlaK, A pair of dual integral equations occurring in diffraction theory, Proc. Edinburgh Math. Soc. (2) 13 (1962), 179-188.

(2) I. N. SNEDdon, Mixed boundary value problems in potential theory (NorthHolland, 1966).

(3) R. P. Srrvastav, On certain integral equations of convolution type with Bessel function kernels, Proc. Edinburgh Math. Soc. (2) 15 (1966), 111-116.

UNIVERSTTY OF STRATHCLYDE

\section{GLASGOW}

